\title{
Efficient series solutions for non-linear flow over topography
}

\section{S. R. Belward W. W. Read P. J. Higgins*}

(Received 1 June 2001; revised 30 September 2002)

\begin{abstract}
Fluid flowing over topography occurs in many physical situations. As a consequence, study of flow over topography has been a research topic of prime interest for many decades. Formally, the problem can be modelled as a nonlinear free boundary problem. Although methods such as boundary integrals are typically used, analytic series methods have also been developed to solve some of these problems. Arguably the hardest problem to solve is the lee wave problem: when the flow conditions are suitable, waves form downstream of the obstacle. Wave solutions pose several problems for the analytic series methods. The solution method is iterative, and at each step the existing solution must be updated. For the iterative scheme to converge, very accurate series solutions must be obtained at each step. The convergence rate
\end{abstract}

*School of Mathematical and Physical Sciences, James Cook University, Townsville, Australia. mailto:shaun.belward@jcu.edu.au ${ }^{0}$ See http://anziamj.austms.org.au/V44/CTAC2001/Belw for this article, (c) Austral. Mathematical Soc. 2003. Published 1 April 2003. ISSN 1446-8735 
of the series solution itself is critical in this process, and depends to a large extent on the free boundary representation. In this paper, we compare and discuss the convergence rates for a variety of free surface representations. We show that spectral convergence is possible if the correct representation is used.

\section{Contents}

1 Introduction

C97

2 Problem formulation

C99

3 Solution procedure

C102

4 Convergence of series

C104

5 Discussion and summary

C112

References

C112

\section{Introduction}

The volume of material written on fully nonlinear flow over topography has increased rapidly over the last two decades. In particular the case in which the fluid is inviscid, incompressible and flows without rotation in two dimensions has been solved using many different techniques. These include complex variable methods. For example, Forbes [5] considered flow over a semi-elliptical obstacle, Forbes and Schwartz [6] considered flow over a semi-circle and Dias and Van den Broeck [4] studied flow over a triangular weir. In each 
of these cases conformal mappings specific to the topography under consideration were used. In the early 1990s boundary integral methods in the original variables were developed. These allowed arbitrary topography to be considered, see for example the work of Belward and Forbes [1, 2]. King and Bloor [8] used a generalisation of the Schwarz-Christoffel transformation to solve the same problem. More recently series solution approaches have been developed for this problem, see Read, Belward and Higgins [9].

We assume that the fluid is inviscid, incompressible and flows without rotation so the problem reduces to solving Laplace's equation. Boundary conditions are available, but a complication is that the location of the upper boundary (the free surface) of the fluid is unknown. Thus the boundary conditions on this surface are nonlinear. The series solution and boundary integral approaches mentioned above overcome this problem by guessing the location of the free surface, evaluating a cost function and then updating the location of the free surface on the basis of the behaviour of the cost function. In each case, given a sufficiently accurate initial estimate of the location of the free surface the iterative procedure in use converges to the true location of the free surface.

The series solution approach uses separation of variables. This has the advantage that exact error estimates are immediately available, see Gill, Read and Sneddon [7]. The basis functions used satisfy the partial differential equation and boundary conditions on two sides of the computational domain exactly. Once the basis functions are determined, the efficiency of the algorithm depends on the update method employed, the computation of the coefficients in the series expansions and the convergence rate of the series.

In this paper we concentrate on the latter. We show that the convergence rate of the series depends crucially on the way the location of the free surface is approximated. In particular, if the 
representation of the free surface takes special account of the basis functions for the problem, then spectral convergence of the series solution of the partial differential equation is observed.

In Section 2 the problem is mathematically formulated and in Section 3 the analytical series solution method is presented. The convergence rate of the series is discussed in Section 4 with results for differing representations of the free surface. Finally in Section 5 results are discussed and summarised.

\section{Problem formulation}

We consider irrotational flow of a single layer of inviscid, incompressible, fluid of constant density over arbitrary topography. Far upstream of the obstacle the fluid flows with uniform height, $H$, and with velocity $c \mathbf{i}$. Variables in the problem are nondimensionalised with respect to $H$ and $c$ and we work purely in a nondimensional system. The profile of the obstacle is $y=B(x)$ where $x$ and $y$ are horizontal and vertical coordinates respectively. The surface of the fluid is denoted by $y=\eta(x)$, this is unknown at the outset. Only flow stationary with respect to the obstacle is considered, so partial derivatives with respect to time are identically zero. The fluid system after nondimensionalisation is shown schematically in Figure 1.

Following nondimensionalisation a dimensionless parameter results, the Froude number, defined as $F=c / \sqrt{g H}$ in which $g$ is the acceleration due to gravity. The characteristics of the flow are largely dependent on the value of $F$. In this work we consider only subcritical flows which means $F<1$. These flows are characterised by a region of almost uniform flow upstream of the obstacle with a train of "lee waves" downstream of the obstacle. This is the most 


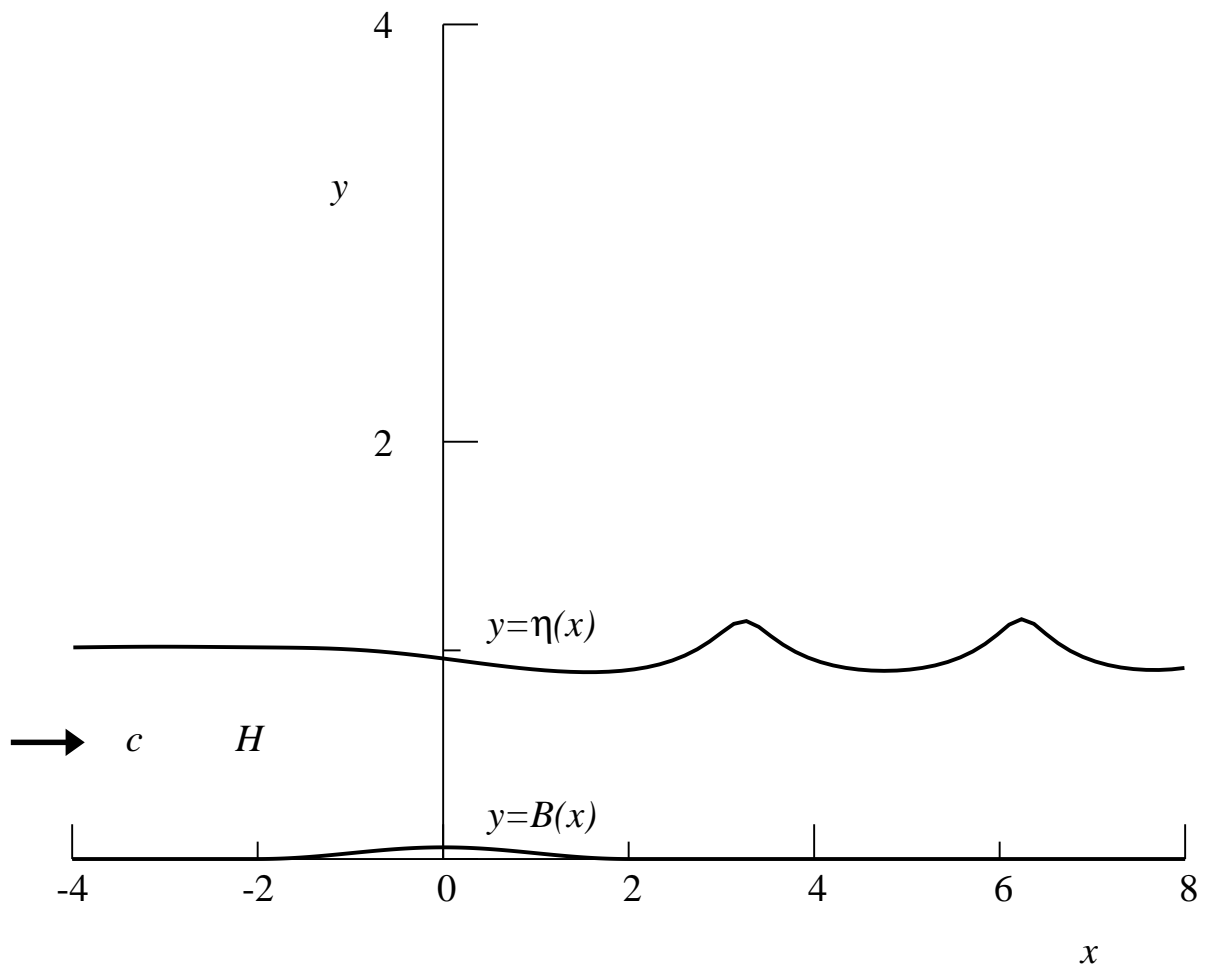

FiguRE 1: A schematic representation of the problem 
difficult region of the parameter space in which to find solutions.

Let $\Psi$ be the stream function so that the velocity vector is $\mathbf{q}=$ $(\partial \Psi / \partial y,-\partial \Psi / \partial x)$ then fluid incompressibility yields

$$
\nabla^{2} \Psi(x, y)=0 .
$$

No fluid penetrates the bottom surface:

$$
\Psi(x, y)=0 \quad \text { on } \quad y=B(x) .
$$

No fluid leaves the flow, so the top surface is a streamline:

$$
\Psi(x, y)=1 \quad \text { on } \quad y=\eta(x) .
$$

The momentum equation is used in its integrated form, the Bernoulli equation, which evaluated on the top surface yields

$$
\frac{1}{2} F^{2}\left[\left(\frac{\partial \Psi}{\partial x}\right)^{2}+\left(\frac{\partial \Psi}{\partial y}\right)^{2}\right]+\eta(x)=\frac{1}{2} F^{2}+1 \quad \text { on } \quad y=\eta(x) .
$$

The upstream conditions are:

$$
\mathbf{q} \rightarrow \mathbf{i}, \quad \eta(x) \rightarrow 1 \quad \text { as } \quad x \rightarrow-\infty .
$$

The downstream conditions are:

$$
\mathbf{q}, \eta(x) \text { are bounded as } \quad x \rightarrow \infty .
$$

Equations (1-6) are to be solved. To apply a series solution technique, the flow domain is truncated to a finite subset of the $x$-axis, $x \in[-L, L]$, where $L$ is chosen large enough so that flow quantities vary slowly with $x$ near $x= \pm L$. We impose the upstream condition (5) with

$$
\mathbf{q}=\mathbf{i} \quad \Rightarrow \quad \Psi(x, y)=y \quad \text { on } \quad x=-L .
$$


Equation (6) is interpreted using

$$
\mathbf{q}=a \mathbf{i} \quad \Rightarrow \quad \Psi(x, y)=a y \quad \text { on } \quad x=L
$$

where $a$ is a constant. The solution procedure is simplified if homogeneous boundary conditions exist at $x= \pm L$. Thus we transform the stream function $\Psi$ to a related function $\psi$ :

$$
\Psi(x, y)=\psi(x, y)+y+\frac{(x+L)}{2 L}(a-1) y .
$$

The problem then reduces to solving

$$
\nabla^{2} \psi(x, y)=0
$$

subject to the homogeneous side boundary conditions

$$
\psi(-L, y)=\psi(L, y)=0
$$

and

$$
\begin{aligned}
\psi[x, B(x)] & =-B(x)-\frac{(x+L)}{2 L}(a-1) B(x)=h^{b}(x), \\
\psi[x, \eta(x)] & =1-\eta(x)-\frac{(x+L)}{2 L}(a-1) \eta(x)=h^{t}(x) .
\end{aligned}
$$

Equations (7-10) represent the transformed problem. Equation (4) is used as a cost function.

\section{Solution procedure}

The solution procedure consists of iteratively updating an initial estimate of the free boundary location (Read, Belward and Higgins [9]). We present only the essential ideas, and those that differ from the previous publication. The series solution is

$$
\psi(x, y)=\sum_{n=1}^{\infty}\left[\alpha_{n} u_{n}(x, y)+\beta_{n} v_{n}(x, y)\right]
$$


where

$$
\begin{aligned}
& u_{n}(x, y)=\sinh \left(\frac{n \pi y}{2 L}\right) \sin \left(\frac{n \pi(x+L)}{2 L}\right), \\
& v_{n}(x, y)=\cosh \left(\frac{n \pi y}{2 L}\right) \sin \left(\frac{n \pi(x+L)}{2 L}\right) .
\end{aligned}
$$

Thus $\psi(x, y)$ satisfies Laplace's equation (7) and the side boundary conditions (8). The constants $\alpha_{n}$ and $\beta_{n}$ are determined using the upper and lower boundary conditions $(9,10)$. Here

$$
\begin{aligned}
h^{b}(x) & =\sum_{n=1}^{\infty}\left[\alpha_{n} u_{n}^{b}(x)+\beta_{n} v_{n}^{b}(x)\right], \\
h^{t}(x) & =\sum_{n=1}^{\infty}\left[\alpha_{n} u_{n}^{t}(x)+\beta_{n} v_{n}^{t}(x)\right],
\end{aligned}
$$

where $u_{n}^{b}(x)=u_{n}[x, B(x)], u_{n}^{t}(x)=u_{n}[x, \eta(x)]$, etc.

Consider the top boundary. We assume that

$$
u_{n}^{t}(x)=\sinh \left(\frac{n \pi \eta(x)}{2 L}\right) \sin \left(\frac{n \pi(x+L)}{2 L}\right)
$$

form a complete linearly independent set of vectors so that

$$
v_{n}^{t}(x)=\sum_{i=1}^{\infty} k_{i n}^{t} u_{i}^{t},
$$

for some constants $k_{i n}^{t}$. Also

$$
h^{t}(x)=1-\eta(x)-\frac{(x+L)}{2 L}(a-1) \eta(x)=\sum_{n=1}^{\infty} \gamma_{n} u_{n}^{t}(x)
$$

for some constants $\gamma_{n}$. 
Using equations (11) and (12) it is possible to solve for $\alpha_{n}$ and $\beta_{n}$. In practice, the series solution is truncated after a sufficient number of terms have been included. We then use

$$
\psi(x, y) \approx \psi_{N}(x, y)=\sum_{n=1}^{N}\left[a_{n} u_{n}(x, y)+b_{n} v_{n}(x, y)\right],
$$

where $\psi_{N}, a_{n}$ and $b_{n}$ are estimators of $\psi, \alpha_{n}$ and $\beta_{n}$ respectively.

The iterative scheme is outlined in Read, Belward and Higgins [9]. The error in the series solution for $\Psi(x, y)$ anywhere in the domain is determined using root mean square (r.m.s.) errors in the upper and lower boundary approximations. For example the error in the approximation to the upper boundary is

$$
\epsilon_{N}^{\eta}=\left[\frac{1}{2 L} \int_{-L}^{L}\left[1-\Psi_{N}(x, \eta(x))\right]^{2} d x\right]^{1 / 2} .
$$

The Bernoulli equation (4) provides a cost function that determines how close the current estimate, $\eta(x)$, is to the true location of the free surface for the flow under consideration. This cost function forms the basis of a quasi-Newton update method for $\eta(x)$.

\section{Convergence of series}

Note that we have left unanswered the question of how the location of the free surface is approximated. Read, Belward and Higgins [9] claimed that the "series solution method does not depend on the method used to represent any of the boundary geometries, including the free boundary." This is true to certain extent; however, the rate of convergence of the series representation $\Psi_{N}$ to $\Psi$ is crucially dependent upon the representation of the free surface. Given that 
hundreds of iterations are required to find any flow solution, and that at each iteration a series solution needs to be found, considerable savings will result if the series converges quickly. We proceed to give strong evidence showing that spectral convergence of the solution at each iteration will occur if the correct representation of the free surface is used.

In order to compare different representations of the free surface we have chosen a cosine shaped obstacle with maximum height $B_{0}$ and base width $d$ :

$$
B(x)=\left\{\begin{array}{cl}
\frac{B_{0}}{2}\left(1+\cos \left[\frac{2 \pi x}{d}\right]\right), & -d / 2 \leq x \leq d / 2, \\
0, & \text { otherwise }
\end{array}\right.
$$

A typical free surface profile is shown in Figure 2 for the case where $F=0.5, B_{0}=0.1$ and $d=4$. Note this compares favourably with solutions found using the method in Belward and Forbes [1], so we are confident with the validity of the solutions computed using the series solution approach.

For the location of the free surface $\eta(x)$ six different methods of approximation were used: linear, quadratic and cubic interpolation, Fourier series, Fourier sine series and Fourier cosine series. Note these approximations were used on the function $h_{t}(x)$ so the representation of $\eta(x)$ is implicit through equation (13).

Figures 3 and 4 show the r.m.s. errors in equations (2) and (3) respectively as a function of the number of terms in the series for each of the six methods. Clearly in both cases the Fourier sine series representation results in superior convergence of the series $\Psi_{N}(x, y)$, followed by cubic spline interpolation. Of surprise here is the speed at which $\Psi_{N}$ converges when a Fourier sine series is used in comparison to the rate $\Psi_{N}$ converges when other Fourier series approximations are used. These differences are highlighted in Figure 5 where 


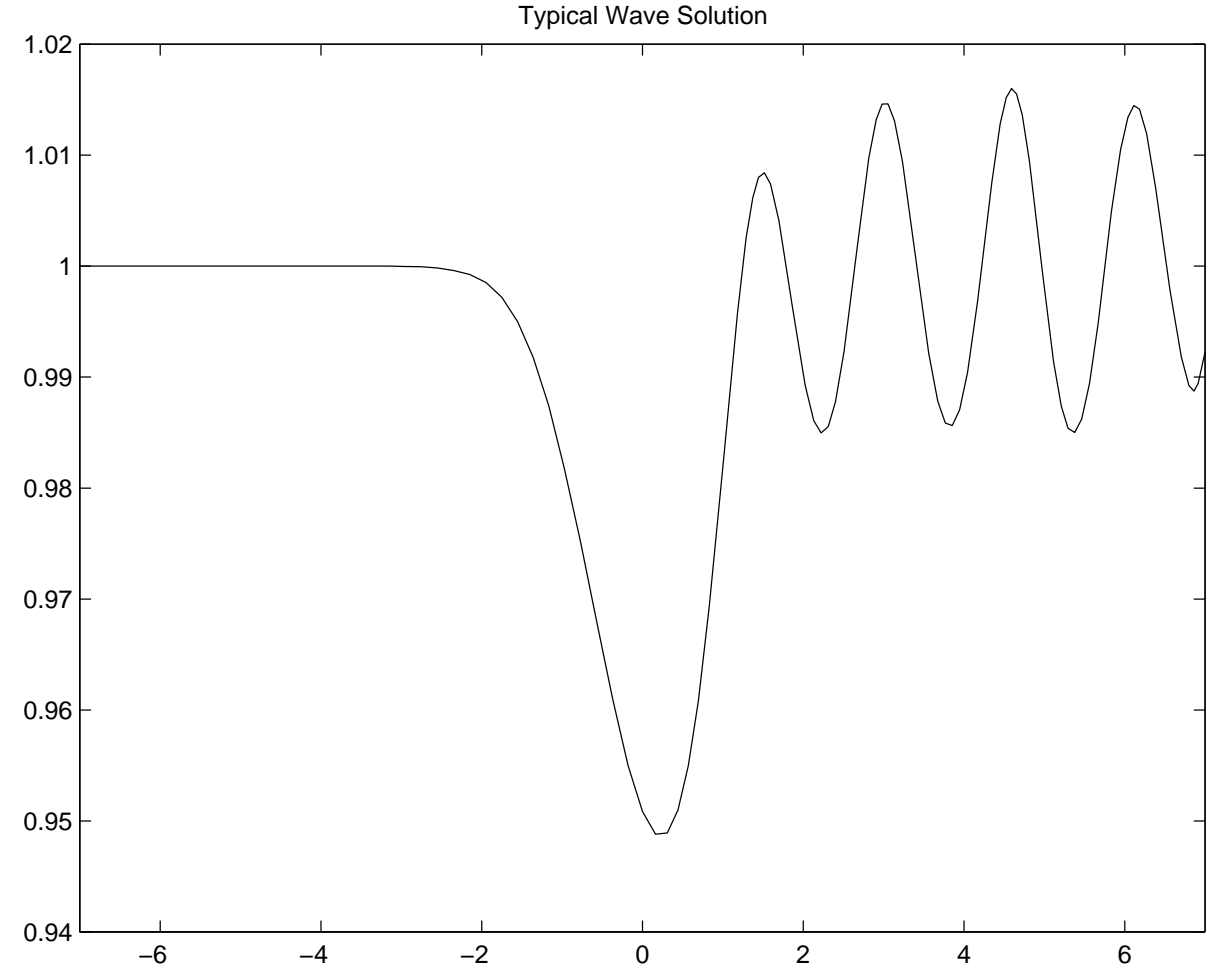

Figure 2: A typical free surface profile. 


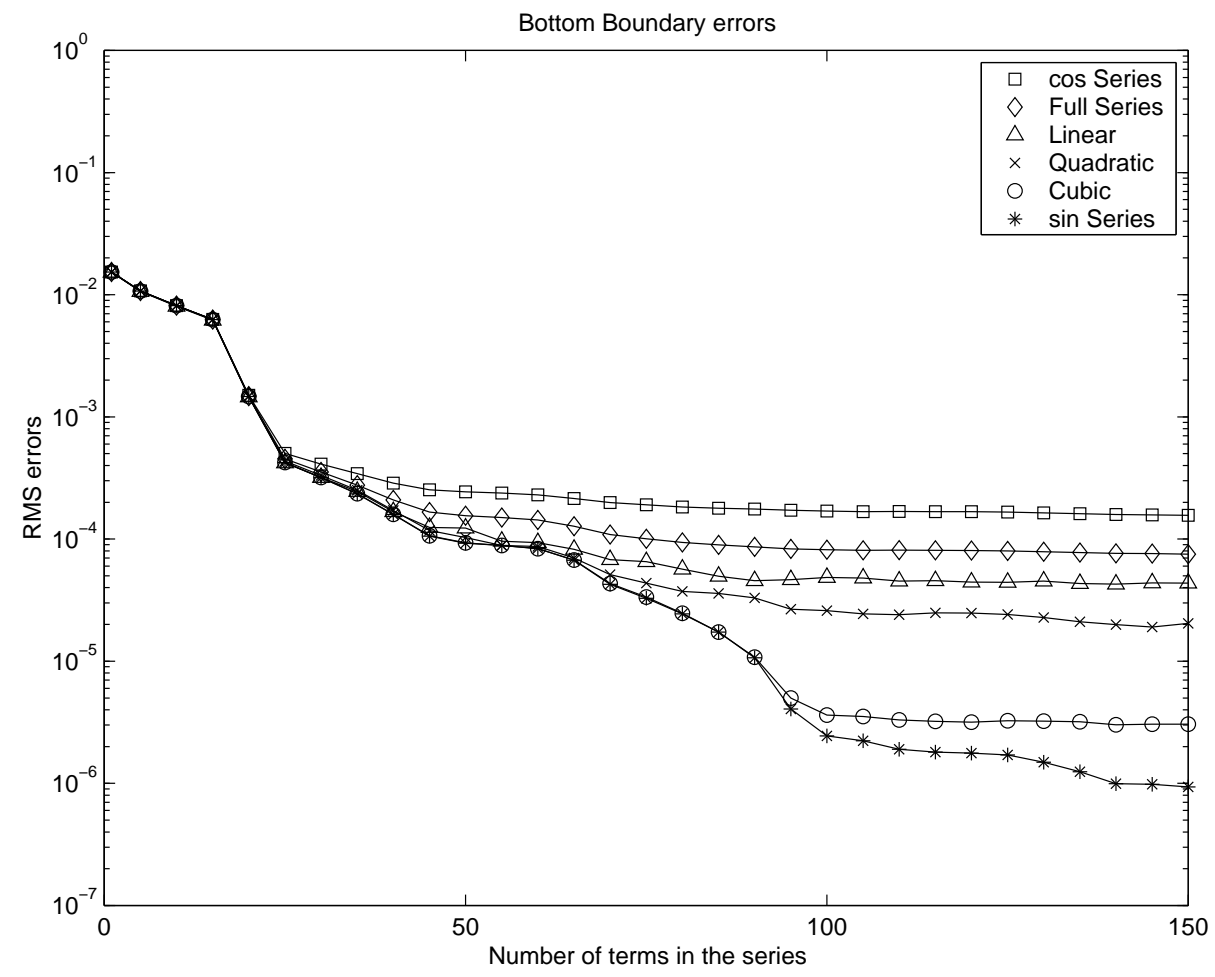

Figure 3: Bottom boundary errors.

r.m.s. errors in $\Psi$ along the top boundary (equation (3)) as a function of the number of terms in the series are presented on a log-log plot for both the Fourier sine and cosine series representations.

Assuming the error takes the form

$$
\epsilon=n^{-k}, \quad \text { so that } \quad \ln \epsilon=-k \ln n,
$$

the log-log plot shows that $k$ is at least 14 when the Fourier sine series representation is used. We believe that the slope on this curve will continue to steepen as more terms in the series are used, and that we have observed spectral convergence. 


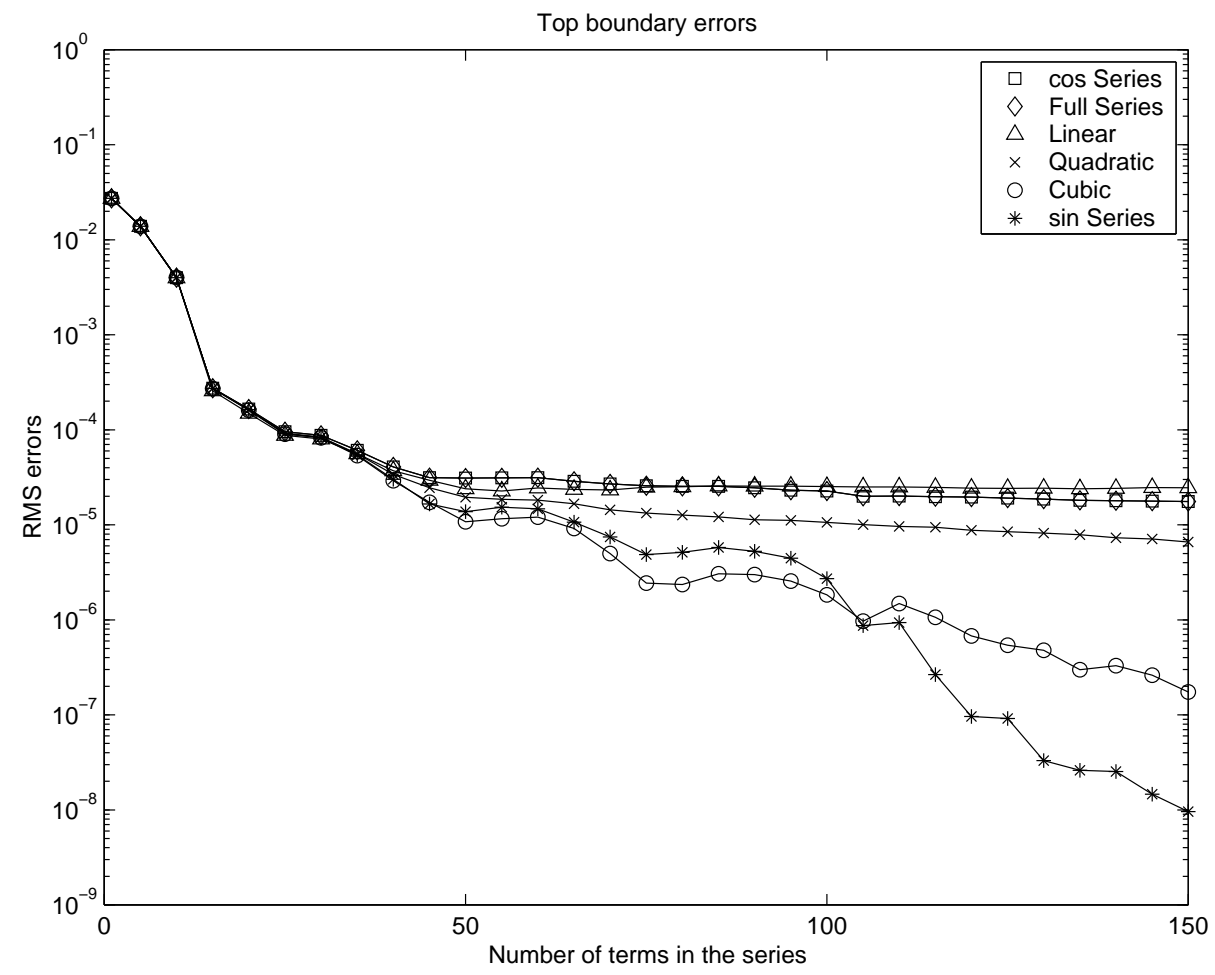

Figure 4: Top boundary errors. 


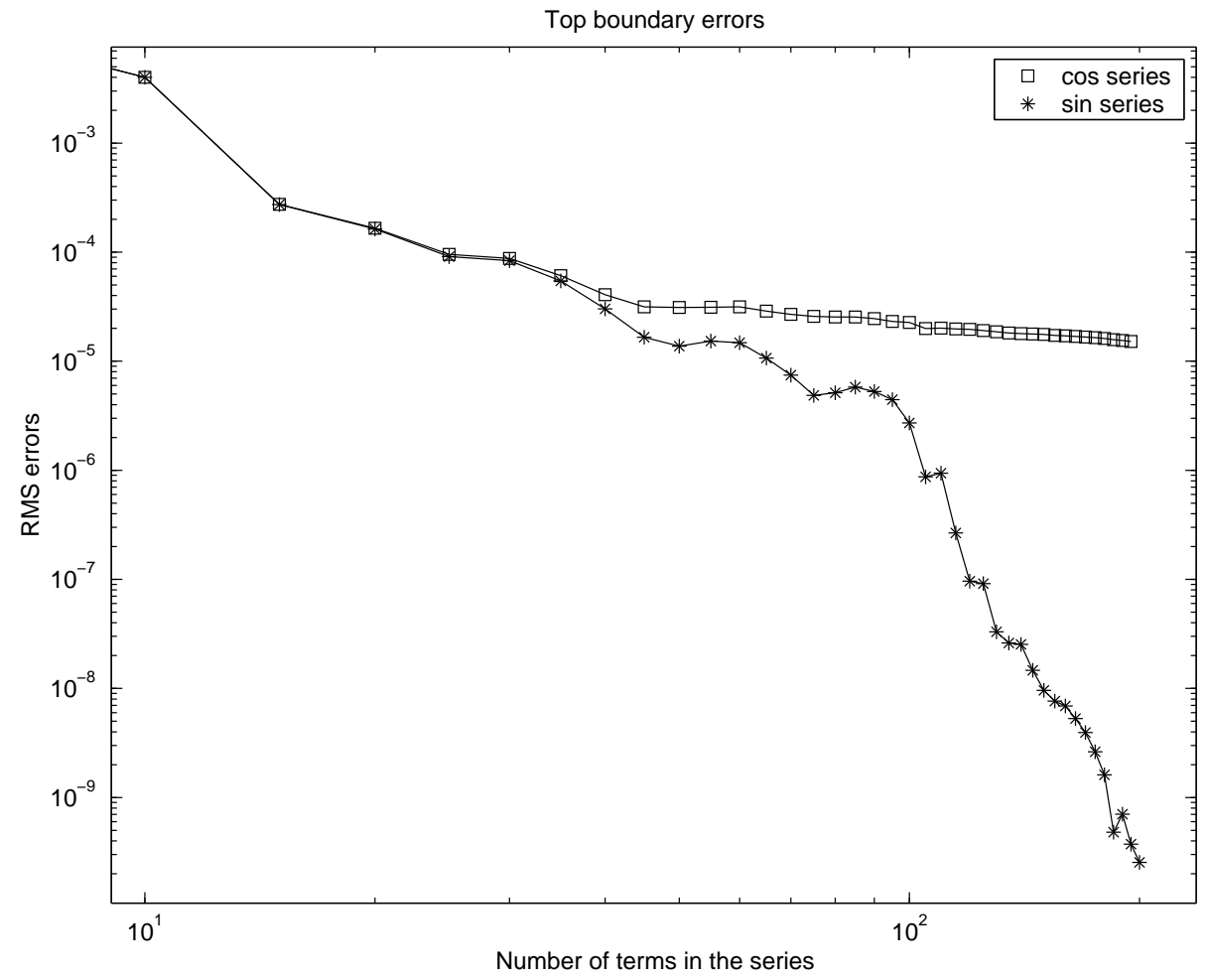

Figure 5: Comparison of sine and cosine series errors on the top boundary. 
A variation of a result presented in Canuto et al. [3] offers an explanation of this behaviour. The basis functions for the series solution of the partial differential equation are clearly non-orthogonal. However, observe that they involve sine functions so intuition leads us to expect that a trigonometric representation of the free surface may present better results than other representations.

Suppose that a Fourier cosine series is used to represent $h_{t}(x)$ over the interval $[-L, L]$. Then

$h_{t}(x)=\frac{a_{0}}{2}+\sum_{n=1}^{\infty} a_{n} \cos \frac{n \pi x}{L} \quad$ where $\quad a_{n}=\frac{1}{L} \int_{-L}^{L} h_{t}(x) \cos \frac{n \pi x}{L} d x$.

Here $h_{t}(-L)=h_{t}(L)=0$ so that using integration by parts gives

$$
\begin{aligned}
a_{n} & =\frac{1}{n \pi}\left[h_{t}(x) \sin \frac{n \pi x}{L}\right]_{-L}^{L}-\frac{1}{n \pi} \int_{-L}^{L} h_{t}^{\prime}(x) \sin \frac{n \pi x}{L} d x \\
& =\frac{L}{n^{2} \pi^{2}}\left[h_{t}^{\prime}(x) \cos \frac{n \pi x}{L}\right]_{-L}^{L}-\frac{L}{n^{2} \pi^{2}} \int_{-L}^{L} h_{t}^{\prime \prime}(x) \cos \frac{n \pi x}{L} d x \\
& =\frac{L}{n^{2} \pi^{2}} \cos n \pi\left(h_{t}^{\prime}(L)-h_{t}^{\prime}(-L)\right)+\frac{L^{2}}{n^{3} \pi^{3}} \int_{-L}^{L} h_{t}^{\prime \prime \prime}(x) \sin \frac{n \pi x}{L} d x .
\end{aligned}
$$

See from this that the coefficients in the Fourier cosine series are small if the first odd derivatives of $h_{t}(x)$ are equal at the end-points of the interval. The size of the coefficients will be determined by the first odd derivative not to satisfy this requirement. Therefore, we would expect to obtain fast convergence using a Fourier cosine series if many of the odd derivatives satisfy this requirement. Given the results such as those displayed in Figures 3, 4 and 5, this is not the case here.

Now suppose that a Fourier sine series is used to represent $h_{t}(x)$ over the interval $[-L, L]$. Then

$$
h_{t}(x)=\sum_{n=1}^{\infty} b_{n} \sin \frac{n \pi x}{L} \quad \text { where } \quad b_{n}=\frac{1}{L} \int_{-L}^{L} h_{t}(x) \sin \frac{n \pi x}{L} d x .
$$


Here $h_{t}(-L)=h_{t}(L)=0$, so that using integration by parts gives

$$
\begin{aligned}
b_{n} & =-\frac{1}{n \pi}\left[h_{t}(x) \cos \frac{n \pi x}{L}\right]_{-L}^{L}+\frac{1}{n \pi} \int_{-L}^{L} h_{t}^{\prime}(x) \cos \frac{n \pi x}{L} d x \\
& =\frac{L}{n^{2} \pi^{2}}\left[h_{t}^{\prime}(x) \sin \frac{n \pi x}{L}\right]_{-L}^{L}-\frac{L}{n^{2} \pi^{2}} \int_{-L}^{L} h_{t}^{\prime \prime}(x) \sin \frac{n \pi x}{L} d x \\
& =\frac{L^{2}}{n^{3} \pi^{3}}\left[h_{t}^{\prime \prime}(x) \cos \frac{n \pi x}{L}\right]_{-L}^{L}-\frac{L^{2}}{n^{3} \pi^{3}} \int_{-L}^{L} h_{t}^{\prime \prime \prime}(x) \cos \frac{n \pi x}{L} d x .
\end{aligned}
$$

See the coefficients in the Fourier sine series are small if many of the first even derivatives of $h_{t}(x)$ are equal at the end-points of the interval. The size of the coefficients is determined by the first even derivative not to satisfy this requirement. Therefore we would obtain fast convergence using a Fourier sine series if the derivatives did satisfy this requirement. Examine Figure 5 to observe that the sine series satisfies equation (14), so that we expect at least the first twelve derivatives of $h_{t}(x)$ to be equal at the end-points (and probably the rest too). Also note that this rate of convergence is not obtained until $n>100$ (approximately). Canuto et al. [3] report that convergence behaviour such as this is typical in the case where derivatives are equal at the end-points; that is, the ultimate rate of spectral convergence is not observed until sufficiently many terms are used in the series.

These results clearly show that the representation of the free surface has a great impact on the convergence rate of the series used to represent $\Psi(x, y)$. In this problem the Fourier sine series gave the best performance. Looking at the basis functions for $\Psi(x, y)$ it is not surprising that this representation for $h_{t}(x)$ works so well. Equation (13) gives many hints that the sine series will give superior performance. 


\section{Discussion and summary}

In this paper we have analysed further the series solution method for nonlinear flow over topography first presented in Read, Belward and Higgins [9]. The method has been applied to what is recognised as one of the most demanding regions of the parameter space, namely that where the Froude number $F<1$ and downstream "lee" waves occur.

We have also used this work as an opportunity to examine the convergence rate of the series used in approximation of $\Psi(x, y)$. We have shown that this is influenced greatly by the representation of the free surface and that if the endpoint derivatives on the upper surface satisfy certain conditions then spectral convergence can be achieved.

\section{References}

[1] S.R. Belward, and L.K. Forbes, "Fully nonlinear two-layer flow over arbitrary topography", J. Eng. Math. 27 (1993) 419-432. C98, C105

[2] S.R. Belward, and L.K. Forbes, "Interfacial waves and hydraulic falls: some applications to atmospheric flows in the lee of mountains", J. Eng. Math. 29 (1995) 161-179. C98

[3] C. Canuto, M.Y. Hussaini, A. Quarteroni, and T.A. Zhang, Spectral Methods in Fluid Dynamics, Springer-Verlag, New York, 1988. C110, C111 
[4] F. Dias and J.-M. Vanden-Broeck, "Open channel flow with submerged obstructions", J. Fluid Mech. 206 (1989) 155-170. C97

[5] L.K. Forbes, "Non-linear, drag-free flow over a submerged semi-elliptical body", J. Eng. Math. 16 (1982) 171-180. C97

[6] L.K. Forbes and L.W. Schwartz, "Free-surface flow over a semi-circular obstruction", J. Fluid Mech. 114 (1982) 299-314. C97

[7] A.W. Gill, W.W. Read and G.E. Sneddon, "On solutions to Laplacian free boundary problems" in Computational techniques and applications: CTAC95 (eds. R.L. May and A.K. Easton), (World Scientific, Singapore, 1996) 343-350. C98

[8] A.C. King and M.I.G. Bloor, "Free surface flow of a stream obstructed by an arbitrary bed topography", Quart. J. Mech. Appl. Math. 43 (1990) 87-106. C98

[9] W.W. Read, S.R. Belward and P.J. Higgins, "Iterative schemes for series solutions to Laplacian free boundary problems" in Computational techniques and applications: CTAC95 (eds. R.L. May and A.K. Easton), (World Scientific, Singapore, 1996) 669-676. C98, C102, C104, C112 